\title{
Readmissão hospitalar por insuficiência cardíaca em um hospital de ensino do interior do estado de São Paulo-SP*
}

\author{
Hospital readmission for heart failure in a teaching hospital \\ in the state of São Paulo-SP
}

Marcio B. Reis ${ }^{1}$, Marina G. Dias ${ }^{1}$, Mariana S. Bibanco' ${ }^{1}$ Camila T. Lopes², Gisela N. Gea ${ }^{3}$

\begin{abstract}
RESUMO
Estudo descritivo retrospectivo de pacientes readmitidos por insuficiência cardíaca crônica, com o objetivo de quantificar as taxas de readmissão hospitalar não planejada em até 30 dias e fatores possivelmente associados à readmissão. As taxas de readmissão hospitalar em 2010 e 2011 foram de 3,91\% e $2,41 \%$, respectivamente, sendo a média de tempo até a readmissão de $14,5 \pm 7,1$ dias. A etiologia mais frequente da insuficiência cardíaca foi a doença valvar, seguida da hipertensão arterial. As mulheres foram mais acometidas e $96 \%$ dos pacientes foram readmitidos em decorrência da persistência ou recorrência do episódio que motivou a admissão inicial. Não foram obtidos dados satisfatórios quanto às possíveis causas que teriam levado à persistência ou recorrência do episódio de doença.
\end{abstract}

Descritores: Insuficiência Cardíaca; Readmissão Hospitalar; Hospitalização.

\begin{abstract}
Retrospective descriptive study of patients readmitted for chronic heart failure, aiming to quantify the rates of unplanned hospital readmission within 30 days and factors possibly associated with readmission. Hospital readmission rates in 2010 and 2011 were $3.91 \%$ and $2.41 \%$, respectively, the average time until readmission being $14.5 \pm 7.1$ days. The most frequent heart failure etiology was valve disease, followed by hypertension. Most were women and $96 \%$ were readmitted as a result of the persistence or recurrence of the episode that prompted the initial admission. No satisfactory data regarding the possible causes of persistence or recurrence of the disease were found.
\end{abstract}

Keywords: Heart Failure; Patient Readmission; Hospitalization.

1. Discente do Curso de Graduação em Medicina da Universidade do Oeste Paulista, Presidente Prudente-SP, Brasil

2. Doutora em Enfermagem, Enfermeira da UTI Adulto do Hospital Universitário da USP, São Paulo-SP, Brasil.

3. Mestre, Docente do Curso de Graduação em Medicina da Universidade do Oeste Paulista, Presidente Prudente-SP, Brasil
Correspondência:

Universidade do Oeste Paulista, Faculdade de Medicina de Presidente Prudente.

Artigo recebido em 18/12/2013 Aprovado para publicação em 20/08/2014

* Trabalho realizado no Departamento de Cardiologia do Hospital Regional de Presidente Prudente-SP, Brasil 


\section{Introdução}

De acordo com a III Diretriz Brasileira de Insuficiência Cardíaca Crônica, no ano de 2007, as doenças cardiovasculares representaram a terceira causa de internações no Sistema Único de Saúde, com 1.156.136 hospitalizações. ${ }^{1}$ A insuficiência cardíaca (IC) - síndrome em que há incapacidade do coração em manter a adequada perfusão tecidual ou fazê-la às custas de altas pressões de enchimento ventricular foi a causa mais frequente de internação por doença cardiovascular. ${ }^{1,2}$ No mesmo ano, foram gastos com esta doença cerca de R \$ 233 milhões. Em Presidente Prudente-SP, foram gastos R \$ 79.494, 59 no ano de 2011, representando $9,02 \%$ do total gasto com doenças cardiovasculares. ${ }^{3}$

O tratamento de doenças crônicas, como a IC, é complexo e inclui aprender a viver com os sintomas, adaptações do estilo de vida e regimes medicamentosos e não medicamentosos, que se destinam a manter os sintomas sob controle e evitar complicações. ${ }^{4}$ A cronicidade da IC alterna entre estabilidade e fases de descompensação aguda, caracterizadas por frequentes reinternações hospitalares. ${ }^{2}$ Entretanto, a adesão à terapêutica de longo prazo para doenças crônicas em países desenvolvidos é, em média, de 50\%. Nos países em desenvolvimento, as taxas são ainda mais baixas. ${ }^{5}$

O envelhecimento da população, em geral conduz a um aumento do número de doenças crônicas e morbidades múltiplas, desencadeando maior demanda por serviços de saúde, especialmente leitos hospitalares. Em decorrência dessa crescente demanda, há sobrecarga dos leitos hospitalares, resultando em alta precoce de pacientes, tendo como consequências elevadas taxas de readmissão. ${ }^{6}$

A não adesão ao tratamento como um reflexo de abordagem não efetiva do paciente ou da complexidade do tratamento, bem como a ocorrência de complicações referentes à internação inicial, pode implicar em readmissões do paciente com IC.

Readmissões não planejadas podem ser consideradas um evento sentinela para questionar a qualidade do cuidado durante e após a estadia inicial. ${ }^{7}$ As taxas de readmissão podem ser usadas como uma medida da eficácia do tratamento hospitalar, sendo utilizadas cada vez mais como um indicador de qualidade de cuidados. ${ }^{8}$ A readmissão hospitalar é definida como a admissão subsequente à internação índice (primeira admissão hospitalar durante o período de seguimento), no intervalo de tempo considerado, independentemente do diagnóstico que o motivou ou do local onde ocorreu a readmissão. ${ }^{9}$

Considera-se que a maior parte das readmissões hospitalares não planejadas potencialmente evitáveis ocorre no primeiro mês após a alta, sendo este, portanto, o período que melhor traduz a qualidade dos cuidados prestados. ${ }^{10)}$ Os critérios de readmissão hospitalar não planejada evitável são: recorrência ou persistência do episódio de doença que motivou a admissão na internação inicial; complicação reconhecida como evitável; readmissão por motivo social ou psicológico, que pode potencialmente ser controlado pelos serviços hospitalares (a adesão inadequada ao esquema terapêutico prescrito após alta pode ser incluída neste critério).

A identificação da taxa de readmissões hospitalares em 30 dias por IC permitirá inferir a qualidade do cuidado prestado. Estes resultados podem ser base para propostas de estratégias que possam identificar e corrigir possíveis falhas do cuidado médico inicial, reduzindo o impacto causado pelas readmissões.

Assim, o objetivo deste estudo foi quantificar as taxas de readmissão hospitalar não planejada em até 30 dias e fatores possivelmente associados à readmissão.

\section{Material e Métodos}

Foi realizado um estudo descritivo retrospectivo, nas dependências do segundo maior hospital estadual do Estado de São Paulo. Trata-se de um hospital-escola geral de grande porte, com 444 leitos, que gera 1700 saídas por mês.

Foram analisados prontuários de pacientes maiores de 18 anos admitidos por IC no hospital citado, e que receberam alta no período de janeiro de 2010 a dezembro de 2011. Os critérios de exclusão foram: alta por óbito; alta por transferência para outras Instituições hospitalares; admissão hospitalar programada nos 30 dias anteriores ao primeiro dia de internação índice; readmissão programada (por exemplo, para a realização de exames em um segundo momento).

Os pacientes foram classificados de acordo com o grau de limitação funcional, conforme descrito nos prontuários. É preconizado pela New York Heart Association (NYHA): classe I (sem nenhuma limitação; atividades físicas não causam fadiga, palpitação ou dispneia indevidas), classe II (pequena limitação; 
paciente fica confortável em repouso; atividades físicas cotidianas causam fadiga, palpitação ou dispneia), classe III (limitação marcante; paciente fica confortável em repouso; sintomas aparecem com atividades mais leves que as habituais) e classe IV (limitação grave; apresenta sintomas em repouso; não consegue desempenhar nenhuma atividade física sem desconforto).

Para o desenvolvimento da pesquisa, foi utilizado um protocolo, preenchido pelos pesquisadores no período de novembro de 2012 a março de 2013, com os dados obtidos dos prontuários de pacientes. O protocolo é composto por quatro domínios: identificação, utilização prévia dos serviços hospitalares, motivos da readmissão e comorbidades.

Em relação à identificação, foram considerados: sexo, data de nascimento, idade, peso, altura, IMC, raça, naturalidade, procedência, data da alta hospitalar, data da readmissão hospitalar, classe funcional da IC, etiologia e fração de ejeção do ventrículo esquerdo. Quanto à utilização prévia dos serviços hospitalares, foram avaliados: número de internações nos últimos três meses e número de internações no último ano.

Os motivos de readmissão investigados foram: falta de adesão à orientação não medicamentosa (restrição hídrica, restrição sódica, controle de peso) ou falta de adesão à prescrição medicamentosa decorrente de dificuldades sociais ou psicológicas.

As comorbidades investigadas foram: anemia, diabetes mellitus, hipertensão arterial, doença coronariana, doença renal crônica, fibrilação atrial, hipotireoidismo, obesidade e síndrome metabólica, por se tratarem de doenças crônicas frequentemente associadas à IC. ${ }^{1}$

O projeto de pesquisa foi submetido ao Comitê de Ética em Pesquisa do hospital e da Universidade do Oeste Paulista, de acordo com a resolução 196/ 96 do Conselho Nacional de Saúde, e aprovado, sob $\mathrm{n}^{\mathrm{o}}$ de protocolo 1270.

Os resultados das variáveis numéricas contínuas (peso, altura, índice de massa corpórea e peptídeo natriurético tipo B) e discretas (idade, fração de ejeção do ventrículo esquerdo, número de internações anteriores) são apresentados como média \pm desvio padrão ou mediana. Foi utilizada estatística descritiva - frequências absolutas e relativas - para apresentar os resultados dos dados qualitativos (classe funcional, sexo, raça, naturalidade, procedência, etiologia, motivo do internação e comorbidades).

\section{Resultados e Discussão}

É importante que a taxa de readmissão em 30 dias seja mensurada para acompanhar o desempenho do hospital na prevenção de complicações pós-alta, na habilidade educacional em transmitir orientações de cuidado pós-alta, além de propiciar uma transição tranquila do âmbito hospitalar para o retorno às atividades cotidianas.

A evolução dos pacientes com IC é dependente de inúmeras variáveis, dentre elas, a forma de apresentação da doença, as características do paciente, a gravidade da doença e o tratamento que vinha recebendo e aquele que receberá durante e após a descompensação cardíaca. ${ }^{13}$

Entre janeiro de 2010 e dezembro de 2011, 1088 pacientes hospitalizados por IC receberam alta no local de investigação, com idade média de 67,9ะ10,6 anos, $54,3 \%$ do sexo feminino. Esses dados coincidem com as internações por IC em 2007 no Brasil, quando $69,8 \%$ dos pacientes tinham 60 anos ou mais, dos quais $50,6 \%$ eram mulheres. ${ }^{1}$

A Tabela 1 apresenta as principais características demográficas e clínicas da população estudada.

A principal etiologia da IC no Brasil é a miocardiopatia isquêmica secundária à hipertensão arterial. ${ }^{1}$ De fato, a hipertensão foi a comorbidade mais prevalente dentre os paciente readmitidos, porém a etiologia mais frequente da IC foi a doença valvar.

A maioria dos pacientes encontrava-se na classe funcional IV, seguida da classe funcional III. Esses dados reforçam a responsabilidade profissional de instrumentalizar os pacientes quanto ao reconhecimento precoce dos sinais e sintomas de descompensação da doença, como edema de membros inferiores e aumento de peso em curto período, tanto no contexto ambulatorial quanto no contexto hospitalar.

A prevalência similar de reinternação de pacientes com fração de ejeção do ventrículo esquerdo preservada e diminuída sugere que a gravidade da evolução da doença possa não ter desempenhado um papel tão importante para a descompensação quanto a adesão às recomendações medicamentosas e não medicamentosas para controle da doença.

O tempo médio de internação foi de 14,4 $\pm 7,1$ dias. Esses resultados são concordantes com a literatura pois, em estudos anteriores, encontram-se valores entre 9,5 e 11 dias. ${ }^{14,15}$

Dentre os 1088 pacientes citados, 4,23\% $(n=46)$ foram readmitidos em até 30 dias por descompensa- 
Tabela 1 - Características demográficas e clínicas dos pacientes readmitidos em até 30 dias por insuficiência cardíaca $(n=46)$. Hospital Universitário do interior do estado de São Paulo-SP, 2010-2011.

\begin{tabular}{lr} 
Variável & $\mathrm{n}(\%)$ \\
\hline Raça & \\
Branca & $24(52,2)$ \\
Parda & $7(15,2)$ \\
Ignorada & $11(23,9)$ \\
Outras & $4(8,7)$ \\
Etiologia da Insuficiência Cardíaca & \\
Cardiopatia isquêmica & $2(4,3)$ \\
Doença valvar & $28(60,9)$ \\
Hipertensão arterial & $20(43,5)$ \\
Outros & $4(8,7)$,
\end{tabular}

Classe funcional New York Heart Association
I
II
III
IV
$3(2,2)$

Fração de Ejeção do Ventrículo Esquerdo

$<50 \%$

$22(47,8)$

$\geq 50 \%$

$24(52,2)$

Comorbidades

Doença renal crônica

Dislipidemias

Anemia

$5(10,9)$

$5(10,9)$

$8(17,4)$

Hipotireoidismo

$10(21,7)$

Fibrilação atrial

$17(37,0)$

Diabetes mellitus

$23(50,0)$

Hipertensão arterial readmissões, e, de modo geral, cerca de 30\% dos pacientes hospitalizados para compensação necessitam ser readmitidos no primeiro ano de seguimento. ${ }^{16} \mathrm{~A}$ gravidade da cardiopatia e o tratamento prescrito têm um papel fundamental nessas readmissões. ${ }^{13}$ Nesse aspecto, nossos dados diferem da literatura, pois $78,2 \%$ dos pacientes necessitaram ser atendidos uma ou mais vezes no primeiro ano de seguimento, sendo que $56,5 \%$ foram readmitidos no primeiro trimestre.

As taxas de readmissões hospitalares para 2010 e 2011 no hospital de investigação foram 3,91\% e $2,41 \%$, respectivamente. Essas taxas foram inferiores àquelas encontradas na maioria dos estudos na literatura, que encontraram taxas que variam de 5 a $25 \% .9,11,12$

O objetivo do tratamento da IC consiste em alcançar e manter a estabilidade dos pacientes. A adesão ao tratamento com o uso regular dos medicamentos e as alterações no estilo de vida são essenciais para evitar crises de descompensação. Isso tem sido uma importante limitação para alguns pacientes, e por conta disso, as taxas de readmissões continuam elevadas. ${ }^{17}$ Nossos dados corroboram esta premissa, pois 96\% dos pacientes foram readmitidos em decorrência da persistência ou recorrência do episódio de doença. Contudo, não foram encontrados registros em prontuários quanto ao motivo da descompensação da doença, como falta de adesão à orientação não medicamentosa ou medicamentosa. A falta de tal informação dificulta a orientação de alta quanto às práticas específicas para manter a doença compensada. A investigação desses fatores, logo, deve ser encorajada.

\section{Conclusão}

A taxa de readmissão hospitalar da insuficiência cardíaca em até 30 dias foi de 4,23\%, em decorrência da persistência ou recorrência do episódio de doença. No entanto, não foram obtidos dados satisfatórios quanto às possíveis causas que teriam levado à descompensação.

A baixa adesão ao tratamento é uma das principais causas de descompensação da IC. Logo, a implementação de estratégias de educação sistemática que se mostrem eficazes é fundamental. Acredita-se que o investimento em cuidados domiciliares, uma política de integração com a rede de atenção primária e retorno ambulatorial precoce possam ser alternativas para evitar reinternações não planejadas.

Pacientes com IC apresentam altos índices de 


\section{Referências}

1. Bocchi EA, Marcondes-Braga FG, Ayub-Ferreira, SM, Rohde LE, Oliveira WA, Almeida DR, et al. Sociedade Brasileira de Cardiologia. III Diretriz Brasileira de Insuficiência Cardíaca Crônica. Arq. Bras. Cardiol. 2009; 93: 1-7.

2. Lopes AC, et al. Tratado de clínica médica, 2 ed. São Paulo: Roca, 2009.

3. Morbidade hospitalar do SUS - Presidente Prudente/SP - Doenças do aparelho circulatório - Insuficiência cardíaca - Dez/2011. Disponível em:

<http://tabnet.datasus.gov.br/cgi/tabcgi.exe?sih/cnv/nisp.def>. Acesso em: 25 mar. 2012

4. Franzen E, Almeida MA, Aliti G, Bercini RR, Menegon DB, Rabelo ER. Adultos e idosos com doenças crônicas: implicações para o cuidado de enfermagem. Rev. HCPA. 2007; 27, 2 : 28-31.

5. World Health Organization. Adherence to long-term therapies: evidence for action. Genebra: World Heart Organization, 2003.

6. Yam CHK, Wong ELY, Chan FWK, Leung MCM, Yeon EK Measuring and preventing potentially avoidable hospital readmissions: a review of the literature. Hong Kong Med J. 2010; 16: 383-9.

7. Laniéce I, Couturier P, Dramé M, Gavazzi G, Lehman S, Jolly $D$, et al. Incidence and main factors associated with early unplanned hospital readmission among French medical inpatients aged 75 and over admitted through emergency units. Age Ageing. 2008; 37: 416-22.

8. Wood JH. Re-admission of elderly patients after in-patient rehabilitation. Ulster Med J. 1996; 65: 142-4.
9. Chambers M, Clarke A. Measuring readmission rates. Br Med J. 1990; 301, p. 1134-6.

10. Benbassat J, Taragin M. Hospital readmissions as a measure of quality of health care. Arch Intern Med. 2000; 160:1074-8.

11. François P, Bertrand D, Beden C, Fauconnier J, Oliv F. Early readmission as an indicator of hospital quality of care. Rev. épidémiol. santé publique. 2001; 49: 183-92.

12. Zamir D, Zamir M, Reitblat T, Zeev W, Polishchuk I. Readmissions to hospital within 30 days of discharge from the internal medicine wards in southern Israel. Eur $\mathrm{J}$ Intern Med. 2006; 17: 20-3.

13. Barreto ACP, Del Carlo CH, Cardoso JN, Morgado PC, Munhoz $\mathrm{RT}$, et al. Re-hospitalizações e morte por insuficiência cardíaca: índices ainda alarmantes. Arq. Bras. Cardiol. 2008; 91: 335-41.

14. Rohde LE, Clausell N, Ribeiro JP, Goldrich L, Netto R, Dec GW. Heath outcomes in decompensated congestive heart failure: a comparison of tertiary in Brazil and United States. Int. J. Cardiol. 2005; 102: 71-7.

15. Villacorta H, Mesquita ET, Cardoso R, Banates T, Maia ER, Silva ACM. Preditores de sobrevida obtidos na unidade de emergência em pacientes atendidos por insuficiência cardíaca descompensada. Rev. Port. Cardiol. 2003; 22: 495-507.

16. Shahar E, Lee S, Kim J, Duval S, Barber C, Luepker RV. Hospitalized heart failure: rates and long-term mortality. J Card Fail. 2004; 10: 374-9.

17. Mangini S, Silveira FS, Silva CP, Grativvol OS, Seguro LFBC, Ferreira SMA, et al. Insuficiência cardíaca descompensada na unidade de emergência de hospital especializado em cardiologia. Arq. Bras. Cardiol. 2008; 90: 433-40. 